\title{
Meropenem-RPX7009 Concentrations in Plasma, Epithelial Lining Fluid, and Alveolar Macrophages of Healthy Adult Subjects
}

\author{
Eric Wenzler, ${ }^{a}$ Mark H. Gotfried, ${ }^{\text {b }}$ Jeffrey S. Loutit, ${ }^{\mathrm{c}}$ Stephanie Durso, ${ }^{\mathrm{c}}$ David C. Griffith, ${ }^{\mathrm{c}}$ Michael N. Dudley, ${ }^{\mathrm{c}} \mathrm{Keith}^{\mathrm{A}}$. Rodvold ${ }^{\mathrm{a}, \mathrm{d}}$ \\ College of Pharmacy, University of Illinois at Chicago, Chicago, Illinois, USA a; Pulmonary Associates, Phoenix, Arizona, USA ${ }^{\text {b }}$; The Medicines Company, San Diego, \\ California, USA'; College of Medicine, University of Illinois at Chicago, Chicago, Illinois, USA ${ }^{\text {d }}$
}

\begin{abstract}
The steady-state concentrations of meropenem and the $\beta$-lactamase inhibitor RPX7009 in plasma, epithelial lining fluid (ELF), and alveolar macrophage (AM) concentrations were obtained in 25 healthy, nonsmoking adult subjects. Subjects received a fixed combination of meropenem ( $2 \mathrm{~g})$ and RPX7009 ( $2 \mathrm{~g})$ administered every $8 \mathrm{~h}$, as a 3-h intravenous infusion, for a total of three doses. A bronchoscopy and bronchoalveolar lavage were performed once in each subject at 1.5, 3.25, 4, 6, or $8 \mathrm{~h}$ after the start of the last infusion. Meropenem and RPX7009 achieved a similar time course and magnitude of concentrations in plasma and ELF. The mean pharmacokinetic parameters \pm the standard deviations of meropenem and RPX7009 determined from serial plasma concentrations were as follows: $C_{\max }=58.2 \pm 10.8$ and $59.0 \pm 8.4 \mu \mathrm{g} / \mathrm{ml}, V_{\mathrm{ss}}=16.3 \pm 2.6$ and $17.6 \pm 2.6 \mathrm{liters} ; \mathrm{CL}=11.1 \pm 2.1$ and 10.1 $\pm 1.9 \mathrm{liters} / \mathrm{h}$, and $t_{1 / 2}=1.03 \pm 0.15$ and $1.27 \pm 0.21 \mathrm{~h}$, respectively. The intrapulmonary penetrations of meropenem and RPX7009 were ca. 63 and 53\%, respectively, based on the area under the concentration-time curve from 0 to $8 \mathrm{~h}\left(\mathrm{AUC}_{0-8}\right)$ values of ELF and total plasma concentrations. When unbound plasma concentrations were considered, ELF penetrations were 65 and 79\% for meropenem and RPX7009, respectively. Meropenem concentrations in AMs were below the quantitative limit of detection, whereas median concentrations of RPX7009 in AMs ranged from 2.35 to $6.94 \mu \mathrm{g} / \mathrm{ml}$. The results from the present study lend support to exploring a fixed combination of meropenem ( $2 \mathrm{~g}$ ) and RPX7009 ( $2 \mathrm{~g}$ ) for the treatment of lower respiratory tract infections caused by meropenem-resistant Gram-negative pathogens susceptible to the combination of meropenem-RPX7009.
\end{abstract}

$T^{\text {he }}$ he prevalence of multidrug-resistant Enterobacteriaceae is increasing worldwide, including those that produce carbapenemases (CRE) (1-4). Carbapenem-resistant Klebsiella spp. and Escherichia coli are estimated to cause 9,300 health care-associated infections each year in the United States (5). The Centers for Disease Control and Prevention recently designated CRE an urgent public health threat requiring aggressive monitoring and prevention strategies for effective patient management (5). Since CRE has become resistant to nearly all available antibiotics (6-8), several $\beta$-lactamase inhibitors are currently in clinical development, including potent inhibitors of the Klebsiella pneumoniae carbapenemase (KPC) enzyme.

RPX7009 is a novel cyclic boronic acid-based $\beta$-lactamase inhibitor that restores the activity of $\beta$-lactams against strains of Enterobacteriaceae that produce serine carbapenemases, particularly the KPC enzyme (9). The combination of meropenem and RPX7009 has demonstrated potent in vitro activity against serine carbapenemases $(10,11)$. A fixed-dose, combination product of meropenem (2 g) and RPX7009 (2 g) (meropenem-RPX7009) is currently in phase 3 clinical investigation for the treatment of complicated urinary tract infections, acute pyelonephritis and serious infections due to CRE, including hospital-acquired and ventilator-associated pneumonia (NCT02166476 and NCT02168946). The dose of RPX7009 is supported by nonclinical toxicology and pharmacokinetic-pharmacodynamic studies, in addition to the safety and pharmacokinetics observed in phase 1 studies in normal healthy subjects where single and multiple doses of RPX7009 were assessed alone and in combination with meropenem $(12,13)$.

The pulmonary penetration of antimicrobials is considered an important factor in their ability to be effective agents in the treatment of lower respiratory tract infections $(14,15)$. Measurement of drug concentrations in epithelial lining fluid (ELF) and alveolar macrophages (AM) has become the most practical, reliable, and reproducible litmus test to gain confidence in the adequate distribution of an antibiotic to the site of infection for the treatment of pneumonia (14-17). The primary objective of this study was to determine and compare the plasma, ELF, and AM concentrations of meropenem and RPX7009 after the intravenous (i.v.) administration of multiple doses of meropenem-RPX7009 to healthy male and female adult subjects. The secondary objective of this study was to assess the safety and tolerability of meropenem-RPX7009 in healthy adult subjects.

(This study was presented in part at the 25th European Congress of Clinical Microbiology and Infectious Diseases, Copenhagen, Denmark, 25 to 28 April 2015.)

\section{MATERIALS AND METHODS}

Study design and subjects. This was a phase I, randomized, open-label, multiple-dose study that evaluated the safety, tolerability, and pharmacokinetics of an intravenous, fixed-dose combination of meropenemRPX7009 (Carbavance; The Medicines Company, Parsippany, NJ) in healthy adults. This study was approved by the Quorum Review Institu-

Received 16 July 2015 Returned for modification 28 August 2015 Accepted 3 September 2015

Accepted manuscript posted online 8 September 2015

Citation Wenzler E, Gotfried MH, Loutit JS, Durso S, Griffith DC, Dudley MN,

Rodvold KA. 2015. Meropenem-RPX7009 concentrations in plasma, epithelial lining fluid, and alveolar macrophages of healthy adult subjects. Antimicrob

Agents Chemother 59:7232-7239. doi:10.1128/AAC.01713-15.

Address correspondence to K. A. Rodvold, kar@uic.edu.

Copyright $\odot$ 2015, American Society for Microbiology. All Rights Reserved. 
tional Review Board and conducted in accordance with Good Clinical Practices at Pulmonary Associates (Phoenix, AZ). Written informed consent was obtained from each subject before study entry.

Inclusion criteria included healthy male or female subjects between 18 and 55 years of age, with body weight between 55 and $100 \mathrm{~kg}$ and a body mass index of 18 to $30 \mathrm{~kg} / \mathrm{m}^{2}$. All subjects demonstrated no clinically significant abnormalities on medical history, vital signs, physical examination, laboratory tests (chemistry, hematology, coagulation, and urinalysis) and 12-lead electrocardiographs prior to study initiation. Subjects must not have used tobacco- or nicotine-containing products within the 6 months preceding study day one.

Exclusion criteria included pregnant or lactating females (male subjects with female partners of childbearing potential were required to remain abstinent or use protocol-defined birth control methods), acute or chronic liver disease, known biliary tract abnormalities, test results positive for HIV antibody, hepatitis B surface antigen, drugs, or alcohol. Subjects with excessive alcohol intake or a history of drug abuse or alcoholism within 2 years prior to enrollment were excluded. Subjects who could not undergo a bronchoalveolar lavage (BAL) due to significant pulmonary or other disease were also excluded. Subjects could not have had a hypersensitivity history or serious adverse reaction to $\beta$-lactam agents or lidocaine. Prescription and nonprescription drugs (including vitamins, herbal, or dietary supplements) were not allowed within 7 days prior to day 1 . Subjects could not have donated blood in excess of $500 \mathrm{ml}$ within a 56-day period or plasma within a 7-day period before study participation. Subjects with a calculated creatinine clearance $<80 \mathrm{ml} / \mathrm{min}$ were excluded (18).

Each subject received i.v. infusions of meropenem (2 g) and RPX7009 $(2 \mathrm{~g})$ administered every $8 \mathrm{~h}$ for a total of three doses. All doses of meropenem-RPX7009 were administered as an i.v. infusion over $3 \mathrm{~h}$. Meropenem-RPX7009 doses were infused via a controlled infusion pump and exact start and stop times of each infusion were recorded. Subjects were confined to the study center for the duration of drug administration and pharmacokinetic sample collection.

Pharmacokinetic samples. Blood samples for the measurement of both meropenem and RPX7009 concentrations were collected before and after the final (third) dose. Sampling times included before (time zero) and $1.5,2.95,3.08,3.25,4,6$, and $8 \mathrm{~h}$ after the start of the final dose. All blood samples $(\sim 6 \mathrm{ml})$ were taken from an indwelling cannula, collected into EDTA tubes, and immediately placed on ice and centrifuged at 3,000 $\times$ $g$ for $10 \mathrm{~min}$ at $4^{\circ} \mathrm{C}$. The supernatant plasma layer was carefully removed and $2 \mathrm{ml}$ of plasma was equally divided between two cryopreservation vials. An equal amount of $1.0 \mathrm{M}$ morpholinepropanesulfonic acid (MOPS) buffer ( $\mathrm{pH} 7.0$ ) was added to the cryovials within $20 \mathrm{~min}$ of centrifugation to equal a 1:1 dilution of plasma to MOPS buffer. The plasma/MOPS aliquots were frozen to $-20^{\circ} \mathrm{C}$ or below until shipment.

Bronchoscopy and bronchoalveolar lavage. Each subject had a single standardized bronchoscopy with BAL in the outpatient facility at $1.5,3.25$, 4,6 , or $8 \mathrm{~h}$ after the start of the last i.v. infusion of meropenem-RPX7009. A total of five subjects were randomly assigned to each BAL sampling time. The sampling times were designed to provide intrapulmonary concentration-time data over the entire dosing interval.

The procedures for bronchoscopy and BAL, and methods of sampling preparation for plasma and intrapulmonary samples have been previously described (17). Briefly, topical lidocaine was applied to the upper airway in preparation for bronchoscopy. A fiber optic bronchoscope (models P-20 or P-20D; Olympus America, Inc., Melville, NY) was inserted into a subsegment of the right middle lobe. Four $50-\mathrm{ml}$ aliquots of sterile $0.9 \%$ normal saline solution were instilled and immediately aspirated and placed in ice. The aspirate from the first $50-\mathrm{ml}$ instillation (BAL-1) was collected and discarded. The aspirates recovered from the second, third, and fourth instillations were pooled (BAL-2). The volume of BAL-2 was measured and recorded and an equal amount of refrigerated MOPS buffer ( $\mathrm{pH}$ 7.0) was added to make a 1:1 dilution. A 4-ml aliquot was removed from BAL-2/MOPS fluid and sent to the laboratory for cell count and differential. A 50-ml aliquot of the BAL-2/MOPS was then centrifuged at $400 \times g$ for $5 \mathrm{~min}$ at $4^{\circ} \mathrm{C}$, and the supernatant (ELF) was immediately separated and placed on dry ice. Additional aliquots of up to $50 \mathrm{ml}$ of the remaining BAL-2/MOPS fluid were added to the pellet (AM), centrifuged, separated, and placed on ice until the entire volume of BAL-2/MOPS was used. The remaining supernatant was separated into three 5-ml aliquots for urea, meropenem, and RPX7009 assay. The cell pellet was also frozen and stored at $-20^{\circ} \mathrm{C}$ or below until shipped to the analytical laboratory for assay of AM concentrations. Subjects were monitored continuously during the bronchoscopy and BAL procedure.

A blood sample to determine plasma urea concentration was obtained just before the scheduled bronchoscopy procedure and was kept on ice until centrifuged. Supernatant plasma was transferred to matrix screwcap tubes and stored at $-20^{\circ} \mathrm{C}$ or below until shipped to the analytical laboratory.

Bioanalytical procedures for determination of meropenem and RPX7009 concentrations. Concentrations of meropenem and RPX7009 in plasma and pulmonary samples were measured by high-performance liquid chromatography-tandem mass spectrometry by MicroConstants, Inc. (San Diego, CA).

The calibration range of the plasma assay for meropenem and RPX7009 was linear $\left(r^{2} \geq 0.999\right)$ from 0.2 to $100 \mu \mathrm{g} / \mathrm{ml}$. A total of 227 unique samples were analyzed in four analytical runs which all met acceptance criteria for standard curve and quality control (QC) samples. The accuracy of the method was determined by comparing the mean measured concentrations with theoretical concentrations of each analyte in the QC samples. The deviation of the mean from theoretical values did not exceed $\pm 2.38 \%$ and $\pm 3.60 \%$ for meropenem and RPX7009, respectively. The precision was determined from the percent coefficient of variation $(\% \mathrm{CV})$ of the QC sample replicates at each concentration level. The \%CV for meropenem and RPX7009 ranged from 2.55 to $5.79 \%$ and 1.64 to $3.3 .1 \%$, respectively.

The calibration range of the assay for pulmonary samples was linear $\left(r^{2}=1.00\right)$ from 0.01 to $5 \mu \mathrm{g} / \mathrm{ml}$ for meropenem and from 0.002 to 1 $\mu \mathrm{g} / \mathrm{ml}$ for RPX7009. A total of 25 unique samples were analyzed in two runs which met acceptance criteria for standard curve and QC samples. Deviation of the mean for meropenem and RPX7009 in ELF did not exceed $\pm 5.67 \%$ and $\pm 1.50 \%$, while the $\% \mathrm{CV}$ was 2.44 to $2.93 \%$ and 2.06 to $3.52 \%$, respectively. Deviation of the mean and \%CV in AM for meropenem and RPX7009 were $\pm 6.67 \%$ and $\pm 4.80 \%$ and 1.56 to $4.95 \%$ and 2.49 to $4.86 \%$, respectively.

Urea concentration determination. The calibration range of the assay for plasma was linear $\left(r^{2} \geq 0.999\right)$ from 2.5 to $5 \mu \mathrm{g} / \mathrm{ml}$. A total of 25 unique samples were analyzed in 2 runs which met acceptance criteria for standard curve and QC samples. Deviation of the mean and \%CV was \pm $10.9 \%$ and 3.52 to $6.24 \%$. The calibration range of the assay for ELF was linear $\left(r^{2} \geq 0.999\right)$ from 0.150 to $2.50 \mu \mathrm{g} / \mathrm{ml}$. A total of 25 samples were analyzed in three runs which met acceptance criteria for standard curve and QC samples. The deviation of the mean and \%CV were \pm 12.3 and 1.91 to $4.44 \%$, respectively.

Calculation of the ELF volume and drug concentrations in ELF and AM. The urea dilution method described by Rennard et al. was used to determine the apparent volume of ELF ( $\left.V_{\mathrm{ELF}}\right)$ (19). Concentrations of meropenem and RPX7009 in ELF were estimated using $C_{\mathrm{ELF}}=C_{\mathrm{BAL}} \times V_{\mathrm{BAL}} / V_{\mathrm{ELF}}$, where $C_{\mathrm{BAL}}$ is the measured concentration of the drug in BAL fluid, $V_{\mathrm{BAL}}$ is the volume of aspirated BAL, $V_{\mathrm{ELF}}$ is the product of $V_{\mathrm{BAL}}$, and the concentration of urea in the BAL fluid in relation to the concentration of urea in plasma.

The concentration of meropenem and RPX7009 in AM was determined by $C_{\mathrm{AM}}=C_{\text {pellet }} / V_{\mathrm{AC}}$, where $C_{\text {pellet }}$ and $V_{\mathrm{AC}}$ are the measured concentration of drug in the pellet and volume of alveolar cells in the cell suspension, respectively. A differential cell count of the BAL fluid was carried out, and the percentage of macrophages was determined. A mean macrophage cell volume of $2.42 \mu \mathrm{l} / 10^{6}$ cells was used in the calculations for $V_{\mathrm{AC}}(20)$. 
TABLE 1 Characteristics of healthy adult subjects receiving meropenem and RPX7009

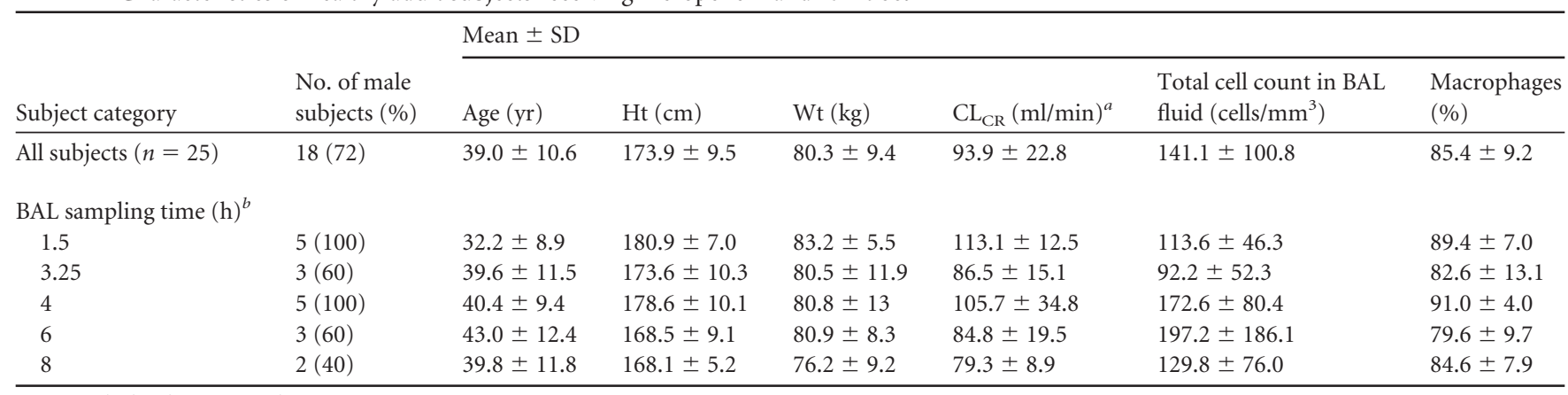

${ }^{a} \mathrm{CL}_{\mathrm{CR}}$, calculated creatinine clearance.

${ }^{b} n=5$ at each bronchoalveolar lavage (BAL) sampling time.

Pharmacokinetic analysis. Noncompartmental analyses (WinNonlin, version 6.3; Pharsight Corp., Cary, NC) were used to generate pharmacokinetic parameters of each subject for meropenem and RPX7009 in plasma. Reported parameters included the peak plasma concentration $\left(C_{\max }\right)$, the volume of distribution at steady-state $\left(V_{\mathrm{ss}}\right)$, the clearance $(\mathrm{CL})$, and the elimination half-life $\left(t_{1 / 2}\right)$. The area under the plasma concentration-time curve over $8 \mathrm{~h}\left(\mathrm{AUC}_{0-8}\right)$ after the last dose of meropenem-RPX7009 was calculated with the linear trapezoidal method. The ratios of ELF and AM concentrations to the simultaneous plasma concentrations were calculated for each subject and summarized for each group at each BAL sampling time. The concentrations at the $8 \mathrm{~h}$ (trough) sampling time were also used as a time zero value for determining the AUC in ELF relative to plasma. Penetration of meropenem and RPX7009 was estimated from the ratios of the $\mathrm{AUC}_{0-8}$ for ELF or AM to the corresponding $\mathrm{AUC}_{0-8}$ in plasma. The $\mathrm{AUC}_{0-8}$ for total plasma concentrations was also transformed to those based on unbound drug concentrations by factoring in plasma protein binding of $2 \%$ for meropenem and $33 \%$ for RPX7009.

Laboratory and safety assessment. Safety was monitored by clinical laboratory tests, physical examination, 12-lead ECGs, vital signs, and monitoring of adverse events. The investigators assessed subjects for the occurrence of adverse events throughout the study. Subjects were tele- phoned after being discharged from the study center to check on their status and inquire about any new or existing adverse events or concomitant medications. An adverse event was defined as any untoward, undesired, unplanned clinical event in the form of signs, symptoms, disease, or laboratory or physical observations, regardless of causal relationship to the study drug.

\section{RESULTS}

Twenty-six healthy adult male and female subjects were enrolled in this study. One subject discontinued the study due to an adverse event of chest discomfort, dizziness, and dyspnea, which were considered by the investigator to be possibly related to study drug. The pharmacokinetic phases for this subject (e.g., blood sample collection to measure drug concentrations in plasma and bronchoscopy with BAL) were not performed.

The characteristics of the 25 study subjects receiving meropenem $(2 \mathrm{~g})$ and RPX7009 $(2 \mathrm{~g})$ every $8 \mathrm{~h}$ for three doses and completing all phases of the pharmacokinetic study are listed in Table 1. The fixed combination of meropenem-RPX7009 was well tolerated in these 25 subjects, and no serious adverse events were
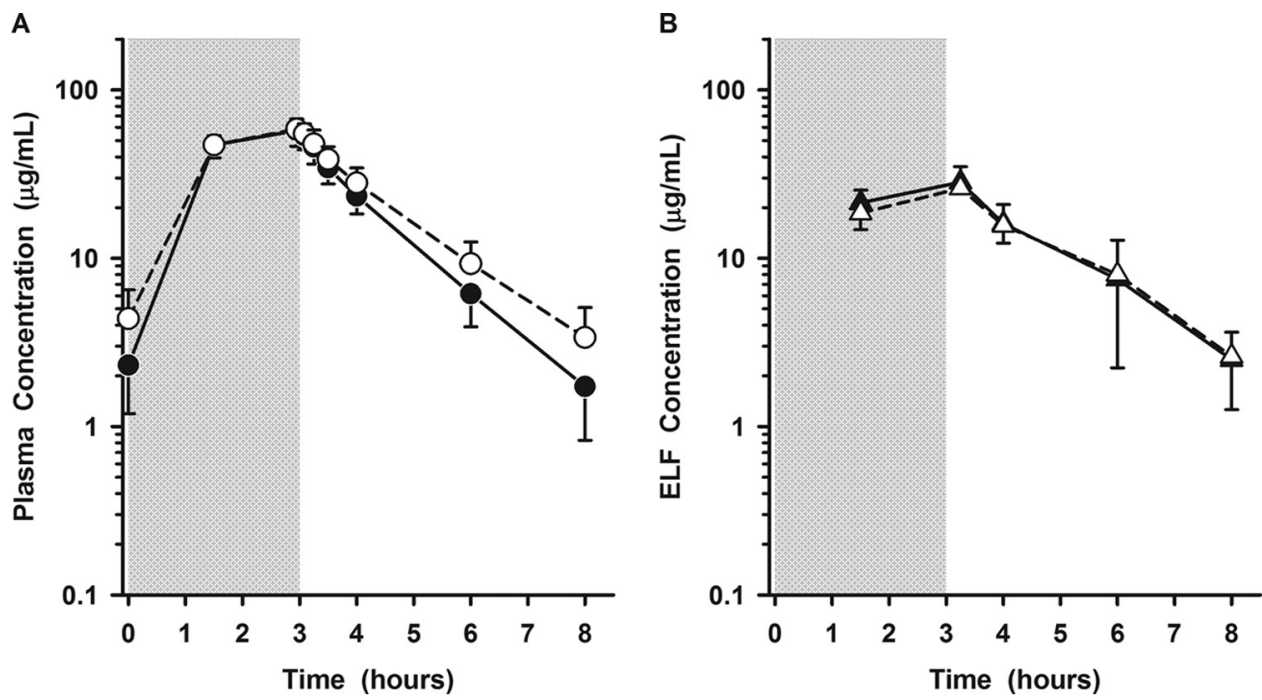

FIG 1 Mean ( \pm the SD) concentration-versus-time profile of meropenem and RPX7009 in plasma (A) and epithelial lining fluid (B) before and after the third dose meropenem (2 g) and RPX7009 (2 g) administered as a 3-h i.v. infusion. In plate A, meropenem is illustrated by the filled circles and a solid line, and RPX7009 is illustrated by open circles and a dashed line. In plate B, meropenem is illustrated by the filled triangles and a solid line, and RPX7009 is illustrated by open triangles and a dashed line. Shaded region represents the 3 -h infusion period. The $y$ axis is in the log scale. 
TABLE 2 Pharmacokinetic parameters of meropenem (2 g) and RPX $(2 \mathrm{~g})$ in plasma infused over $3 \mathrm{~h}$

\begin{tabular}{|c|c|c|c|c|c|}
\hline \multirow[b]{2}{*}{ Treatment } & \multicolumn{5}{|l|}{ Mean $\pm S D$} \\
\hline & $\begin{array}{l}C_{\max } \\
(\mu \mathrm{g} / \mathrm{ml})\end{array}$ & $\begin{array}{l}\mathrm{AUC}_{0-8} \\
(\mu \mathrm{g} \cdot \mathrm{h} / \mathrm{ml})\end{array}$ & $V_{\text {ss }}$ (liters) & $\begin{array}{l}\text { CL } \\
\text { (liters/h) }\end{array}$ & $t_{1 / 2}(\mathrm{~h})$ \\
\hline Meropenem & $58.2 \pm 10.8$ & $186 \pm 33.6$ & $16.3 \pm 2.6$ & $11.1 \pm 2.1$ & $1.03 \pm 0.15$ \\
\hline RPX7009 & $59.0 \pm 8.4$ & $204 \pm 34.6$ & $17.6 \pm 2.6$ & $10.1 \pm 1.9$ & $1.27 \pm 0.21$ \\
\hline
\end{tabular}

reported. One subject experienced treatment-emergent adverse events of headache and vomiting, which were considered to be unrelated to the study drug. No meaningful laboratory, vital sign, ECG, or physical examination findings were observed during the study.

Mean ( \pm the standard deviation [SD]) plasma concentrations of meropenem and RPX7009 before and after the start of the third i.v. 3-h infusion are displayed in Fig. 1A. The mean ( \pm the SD) pharmacokinetic parameters of meropenem and RPX7009 from the serial plasma concentration-time profiles are summarized in Table 2. Mean ( \pm the SD) ELF concentrations of meropenem and RPX7009 after the start of the third i.v. 3-h infusion are displayed in Fig. 1B. The magnitude and time course of meropenem and RPX7009 concentrations in ELF are nearly identical.

The individual concentrations of meropenem in plasma and ELF at the bronchopulmonary lavage sampling times are displayed in Fig. 2A and B, respectively. The mean ( \pm the SD) concentrations of meropenem after the last dose in plasma, ELF, and $\mathrm{AM}$ at the five bronchopulmonary sampling times are reported in Table 3. Mean concentrations of meropenem in plasma and ELF ranged from 1.36 to $41.2 \mu \mathrm{g} / \mathrm{ml}$ and from 2.51 to $28.3 \mu \mathrm{g} / \mathrm{ml}$, respectively. The concentrations of meropenem in the AM were below the quantifiable limit for all samples.

The individual concentrations of RPX7009 in plasma and ELF at the bronchopulmonary sampling times are displayed in Fig. $2 \mathrm{C}$

\section{A}
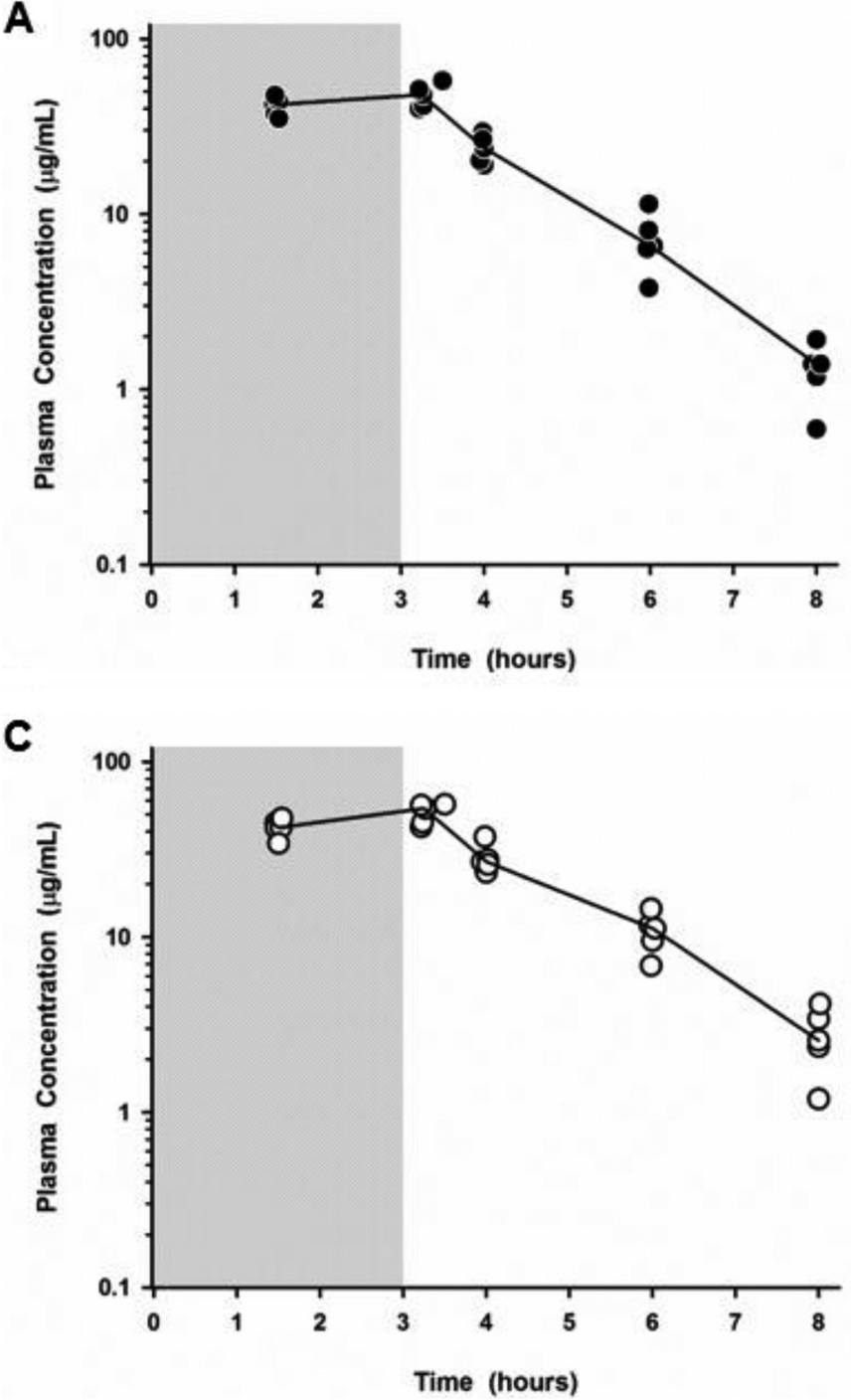

B

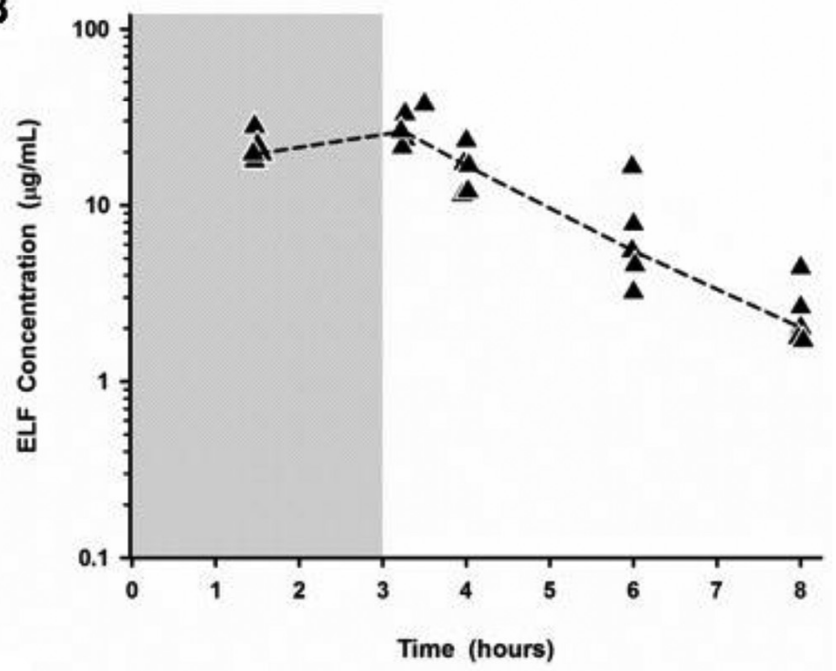

D

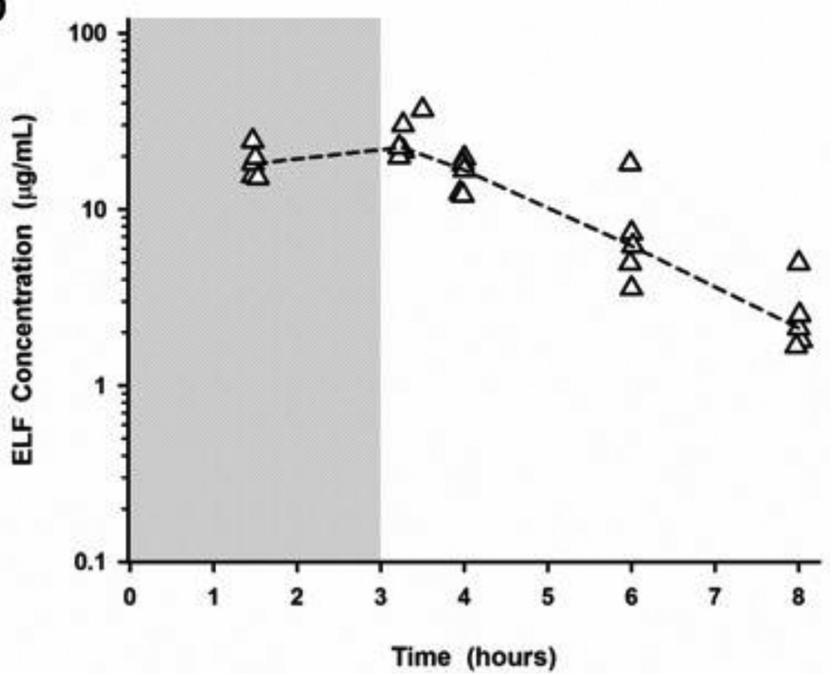

FIG 2 Individual concentrations of meropenem in plasma $(A ;-)$ and epithelial lining fluid (ELF) $(\mathrm{B} ; \mathbf{\Delta})$ and RPX7009 in plasma $(\mathrm{C} ; \mathrm{O})$ and ELF $(\mathrm{D} ; \triangle)$ at 1.5 , $3.25,4,6$, and $8 \mathrm{~h}$ after the third dose of meropenem $(2 \mathrm{~g})$ and RPX7009 $(2 \mathrm{~g})$ administered as a 3 -h i.v. infusion. The shaded region represents the 3-h infusion period. Solid and dashed lines represent the median concentrations in plasma and ELF, respectively. The $y$ axis is in the log scale. 
TABLE 3 Meropenem concentrations in plasma (total), epithelial lining fluid, and alveolar macrophages at sampling time of bronchoscopy and

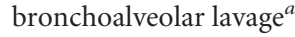

\begin{tabular}{|c|c|c|c|}
\hline \multirow[b]{2}{*}{ BAL sampling time (h) } & \multicolumn{3}{|c|}{$\begin{array}{l}\text { Mean concn }(\mu \mathrm{g} / \mathrm{ml}) \pm S D \text { of meropenem } \\
\text { in: }\end{array}$} \\
\hline & Total plasma & ELF & $\mathrm{AM}$ \\
\hline 1.5 & $41.2 \pm 5.02$ & $21.4 \pm 3.96$ & BQL \\
\hline 3.25 & $47.7 \pm 7.28$ & $28.3 \pm 6.69$ & BQL \\
\hline 4 & $23.8 \pm 4.30$ & $16.1 \pm 4.77$ & BQL \\
\hline 6 & $7.24 \pm 2.79$ & $7.51 \pm 5.29$ & BQL \\
\hline 8 & $1.36 \pm 0.51$ & $2.51 \pm 1.13$ & BQL \\
\hline
\end{tabular}

${ }^{a}$ ELF, epithelial lining fluid; AM, alveolar macrophages; BAL, bronchoalveolar lavage; $\mathrm{BQL}$, below the quantitative limit of detection $(0.01 \mu \mathrm{g} / \mathrm{ml})$.

and $\mathrm{D}$, respectively. The mean $( \pm$ the $\mathrm{SD})$ concentrations of RPX7009 after the last dose in plasma and ELF at the five bronchopulmonary sampling times are reported in Table 4 . The mean concentrations of RPX7009 in plasma and ELF ranged from 2.74 to $51.1 \mu \mathrm{g} / \mathrm{ml}$ and from 2.61 to $26.1 \mu \mathrm{g} / \mathrm{ml}$, respectively.

Alveolar macrophage concentrations of RPX7009 were measurable in all BAL cell samples and ranged from 1.26 to $93.9 \mu \mathrm{g} /$ $\mathrm{ml}$. The individual concentrations of RPX7009 in AM at the bronchopulmonary sampling times are displayed in Fig. 3. The median and range for AM concentrations of RPX7009 at the five bronchopulmonary sampling times are reported in Table 4 .

Figures $4 \mathrm{~A}$ and $\mathrm{B}$ illustrate the time course of mean ( \pm the SD) plasma and ELF concentrations for meropenem and RPX7009, respectively. The mean ratios of ELF to simultaneous plasma concentrations for meropenem during the 8-hour period after drug administration ranged from 0.525 to 2.13 . The $\mathrm{AUC}_{0-8}$ values based on mean and median ELF concentrations were 111.7 and $102.4 \mu \mathrm{g} \cdot \mathrm{h} / \mathrm{ml}$, respectively. The ratio of ELF to total plasma meropenem concentrations based on the mean and median $\mathrm{AUC}_{0_{-8}}$ values were 0.63 and 0.58 , respectively. The ratios of ELF to unbound plasma meropenem concentrations (protein binding $=2 \%)$ based on the mean and median $\mathrm{AUC}_{0_{-}}$values were 0.65 and 0.59 , respectively.

The mean ratios of ELF and AM to simultaneous plasma concentration for RPX7009 during the 8-hour period after drug administration ranged from 0.45 to 1.01 and 0.062 to 2.58 , respectively. The $\mathrm{AUC}_{0-8}$ values based on mean and median ELF concentrations were 105.1 and $96.7 \mu \mathrm{g} \cdot \mathrm{h} / \mathrm{ml}$, respectively. The ratio of ELF to total plasma RPX7009 concentrations based on the mean and median $\mathrm{AUC}_{0-8}$ values were 0.53 and 0.48 , respectively. The ratios of ELF to unbound plasma RPX7009 concentrations (protein binding $=33 \%$ ) based on the mean and median $\mathrm{AUC}_{0-8}$ values were 0.79 and 0.72 , respectively.

\section{DISCUSSION}

The fixed-dose combination of meropenem (2 g)/RPX7009 (2 g) administered every $8 \mathrm{~h}$, as a $3 \mathrm{~h}$ i.v. infusion, demonstrated a similar time course and magnitude between meropenem and RPX7009 concentrations in plasma and ELF. The intrapulmonary penetration of meropenem and RPX7009 based on $\mathrm{AUC}_{0-8}$ values of ELF and total plasma concentrations were ca. 63 and 53\%, respectively. When unbound plasma concentrations were considered, penetration was 65 and 79\% for meropenem and RPX7009, respectively.

The plasma pharmacokinetic parameters observed in the pres-
TABLE 4 RPX7009 concentrations in plasma (total), epithelial lining fluid, and alveolar macrophages at sampling time of bronchoscopy and bronchoalveolar lavage $^{a}$

Mean concn \pm SD or median concn (range) of RPX7009 in $\mu \mathrm{g} / \mathrm{ml}$ in:

\begin{tabular}{llll} 
BAL sampling time $(\mathrm{h})$ & Total plasma & ELF & AM \\
\hline 1.5 & $42.1 \pm 5.00$ & $18.6 \pm 3.76$ & $2.28(1.29-4.69)$ \\
3.25 & $51.1 \pm 6.78$ & $26.1 \pm 7.12$ & $4.68(2.08-25.1)$ \\
4 & $28.2 \pm 5.32$ & $15.7 \pm 3.36$ & $6.94(1.26-8.36)$ \\
6 & $10.8 \pm 2.82$ & $8.04 \pm 5.81$ & $3.85(2.32-95.9)$ \\
8 & $2.74 \pm 1.12$ & $2.61 \pm 1.35$ & $2.35(2.22-11.7)$ \\
\hline
\end{tabular}

${ }^{a}$ ELF, epithelial lining fluid; AM, alveolar macrophages; BAL, bronchoalveolar lavage.

ent study were comparable to previous analyses of single-dose and dose-escalation studies of meropenem-RPX7009 in healthy subjects $(12,13)$. In addition, the plasma concentrations of meropenem were also similar to those previously described for healthy subjects $(21,22)$ administered 3 h i.v. infusions. The fixed-dose combination of meropenem (2 g) and RPX7009 (2 g) administered as a 3-h i.v. infusion was well tolerated and demonstrated a safety profile similar to that seen in other healthy subject studies, as well as in the clinical use of meropenem over several decades $(12,13,23)$.

This study is the first report of the intrapulmonary disposition of RPX7009. After 2-g doses administered every $8 \mathrm{~h}$ as 3-h i.v. infusions, maximum ELF concentrations were observed at the 3.25-h intrapulmonary sampling time and ranged from 19.9 to $36.7 \mu \mathrm{g} / \mathrm{ml}$. The mean ELF concentration of RPX7009 ranged from 2.61 to $26.1 \mu \mathrm{g} / \mathrm{ml}$, with individual ELF concentrations being greater than 1 and $2 \mu \mathrm{g} / \mathrm{ml}$ in 100 and $92 \%$ of subjects, respectively. The individual and median concentrations for RPX7009 and meropenem in ELF were nearly identical (Fig. 2B and D).

The concentrations of RPX7009 were measurable in all alveolar cell samples collected and the median AM concentrations ranged from 2.28 to $6.94 \mu \mathrm{g} / \mathrm{ml}$. Of note, two subjects in the $6 \mathrm{~h}$ BAL sampling time had the highest reported concentrations of

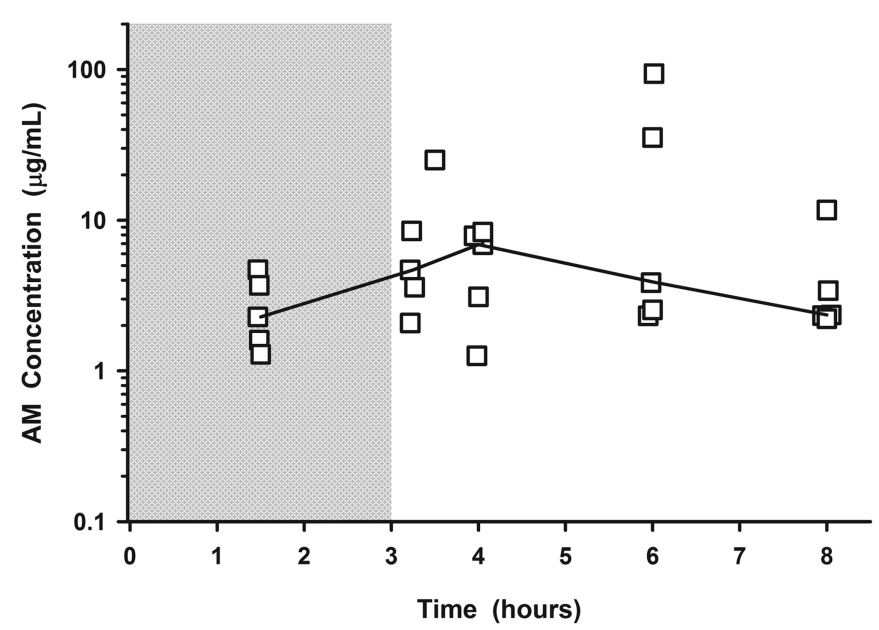

FIG 3 Individual concentrations of RPX7009 in alveolar macrophages (AM) (open squares) at 1.5, 3.25, 4, 6, and $8 \mathrm{~h}$ after the third dose of meropenem (2 g) and RPX7009 (2 g) administered as a 3-h i.v. infusion. The shaded region represents the 3-h infusion period. The solid line represents the median concentration in AM. The $y$ axis is in the log scale. 

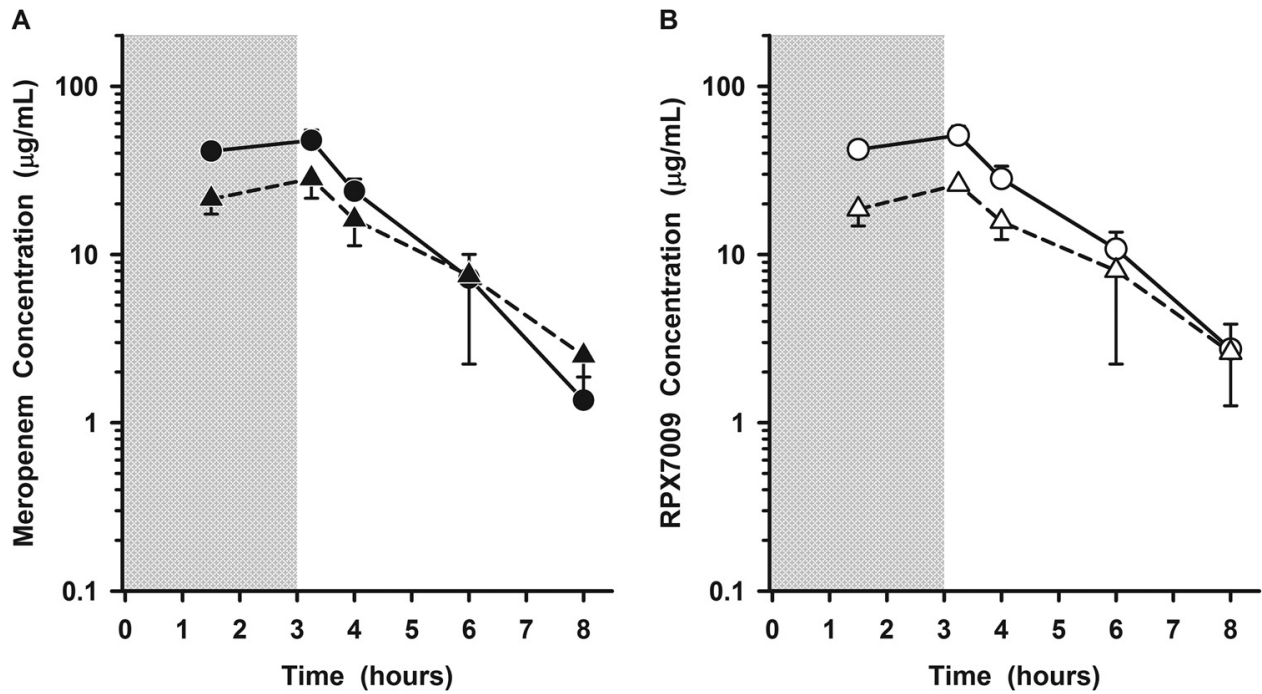

FIG 4 Mean ( \pm the SD) plasma ( $\bigcirc$ and $\bigcirc$; solid line) and epithelial lining fluid (ELF) ( $\Delta$ and $\triangle$; dashed lines) concentration-versus-time profiles of meropenem (A) and RPX7009 (B) at 1.5, 3.25, 4, 6, and $8 \mathrm{~h}$ after the third dose of meropenem (2 g) and RPX7009 (2 g) administered as a 3-h i.v. infusion. The shaded region represents the 3 -h infusion period. The $y$ axis is in the log scale.

RPX7009 in AM (35.4 and $93.9 \mu \mathrm{g} / \mathrm{ml})$ which consequently inflated the mean ( \pm the SD) concentrations of RPX7009 in AM $(27.6 \pm 39.6)$ at this time point. Both of these subjects had extremely high concentrations of red blood cells in their BAL fluid $\left(176,000\right.$ and 226,250 cells $\left./ \mathrm{mm}^{3}\right)$ which may have contributed to such high measurements of AM concentrations (Fig. 3). In contrast, the concentrations of meropenem in the alveolar cells were below the quantifiable limit for all samples. The clinical significance of AM concentrations of the $\beta$-lactamase inhibitor RPX7009 without measurable concentrations of meropenem is unknown.

The concentration-time profile of meropenem in plasma and ELF has been previously studied in healthy subjects. Conte et al. administered meropenem at a dose of $500 \mathrm{mg}, 1 \mathrm{~g}$, or 2 g every $8 \mathrm{~h}$, as 30-min i.v. infusions, for a total of four doses (24). The mean meropenem ELF concentrations at 1, 2, 3, 5, and $8 \mathrm{~h}$ were 5.3, 2.7, $1.9,0.7$, and $0.2 \mu \mathrm{g} / \mathrm{ml}$ for the 500-mg dose and 7.7, 4.0, 1.7, 0.8, and $0.03 \mu \mathrm{g} / \mathrm{ml}$ for the $1-\mathrm{g}$ dose. The ratios of ELF concentrations to total plasma concentrations at the sampling times ranged from 0.49 to 2.3 for the 500 -mg dose and 0.32 to 0.53 for the 1 -g dose. The intrapulmonary penetration of meropenem based on $\mathrm{AUC}_{0-8}$ values of ELF and total plasma concentrations were ca. 43 and $28 \%$ for the $500-\mathrm{mg}$ and 1 -g doses, respectively. For the $2-\mathrm{g}$ dose, the mean meropenem ELF concentrations and penetration ratios at 1 - and 3-h sampling times were 2.9 and $2.8 \mu \mathrm{g} / \mathrm{ml}$, and 0.05 and 0.22 , respectively. For the 2 -g dose, the number of observations were limited $(n=8)$ and calculation of an $\mathrm{AUC}_{0-8}$ value for ELF was not possible.

The findings in our study are not directly comparable to those of Conte et al. due to differences in study design and methodology (24). Our study evaluated a 2 -g dose of meropenem administered as a prolonged infusion over $3 \mathrm{~h}$ and in combination with the $\beta$-lactamase inhibitor RPX7009. In addition, our study included more extensive collection of ELF concentrations $(n=30)$ during the 8-h dosing interval, which allowed a more accurate estimation of the $\mathrm{AUC}_{0-8}$ value. We observed higher mean concentrations of meropenem in both plasma and ELF after prolonged infusion of 2 $\mathrm{g}$ (range, 1.36 to $41.2 \mu \mathrm{g} / \mathrm{ml}$ and 2.51 to $28.3 \mu \mathrm{g} / \mathrm{ml}$, respectively). The mean ratios of ELF to simultaneous plasma concentrations for meropenem during the 8-h period ranged from 0.525 to 2.13. Given more extensive sampling, we are able to characterize penetration more fully than Conte et al. by determining $\mathrm{AUC}_{0-8}$ values for both matrices. The $\mathrm{AUC}_{0-8}$ values based on mean and median ELF concentrations were 111.7 and $102.4 \mu \mathrm{g} \cdot \mathrm{h} / \mathrm{ml}$, respectively. The ratios of ELF to the total plasma meropenem concentrations based on the mean and median $\mathrm{AUC}_{0-8}$ values were 0.63 and 0.58 , respectively.

Several studies have also evaluated plasma and ELF concentrations of meropenem in patients undergoing diagnostic fiber optic bronchoscopy or being treated for severe nosocomial pneumonia (25-27). Plasma and ELF concentrations were measured after a single i.v. dose of meropenem $1 \mathrm{~g}$ infused over $0.5 \mathrm{~h}$ in 30 outpatients undergoing a diagnostic bronchoscopy (25). The mean ratios of ELF to simultaneous plasma concentrations ranged from 0.19 to 1.04 between 0.5 and $6 \mathrm{~h}$ after the meropenem dose. Using the mean values of meropenem concentration at each reported sampling time, our estimation of the $\mathrm{AUC}_{\mathrm{ELF}} / \mathrm{AUC}_{\text {plasma }}$ ratio was $\sim 0.29$ for this study. Lodise et al. described the ELF penetration and population pharmacokinetics of meropenem in adult patients treated for ventilator-associated pneumonia (26). A single ELF concentration was obtained in 17 of 39 patients and the median $\mathrm{AUC}_{\mathrm{ELF}} / \mathrm{AUC}_{\text {plasma }}$ penetration ratio of meropenem was 0.26 based on the mean plasma pharmacokinetic parameters from the population model. Frippiat et al. recently reported a mean $\mathrm{AUC}_{\mathrm{ELF}} / \mathrm{AUC}_{\text {plasma }}$ ratio of 0.29 for meropenem in 25 intensive care unit patients treated for severe nosocomial pneumonia with 3-h infusions of $1 \mathrm{~g}$ every $8 \mathrm{~h}$ (27). This AUC penetration ratio using prolonged infusions was significantly higher $(P=0.047)$ than a value of 0.20 observed in a second group of 30 patients receiving meropenem as 30-min infusions of $1 \mathrm{~g}$ every $8 \mathrm{~h}$. Higher and sustained plasma concentrations may promote enhanced penetration of meropenem into ELF. These findings lend support for the use of extended infusions of meropenem at higher 
doses (e.g., 2 g) to treat patients with severe nosocomial pneumonia $(26,27)$.

The concentration-time profiles and pharmacokinetic parameters of meropenem in the two studies of critically ill patients were markedly different than those observed in our healthy subjects. The large variability and lower plasma concentrations of meropenem observed in patients may be due to an increased apparent volume of distribution and/or wider ranges of meropenem clearance due to altered renal function (e.g., continuous venovenous hemofiltration, augmented renal clearance). This is in contrast to our healthy subjects where the coefficients of variation in distribution and clearance parameters were less than 20\% (Table 2). The meropenem concentrations and $\mathrm{AUC}_{0-8}$ values in ELF of critically ill patients were also notably lower during the 3-h infusion period and more variable after the end of the infusion (see Fig. 1 in Frippiat et al.) (27). In our healthy subjects, the uptake of meropenem into ELF occurred rapidly and tended to follow the same time-dependent profile as plasma concentrations (Fig. 1A and $\mathrm{B})$. The most likely reasons for these observed differences in ELF concentrations in critically ill patients compared to healthy subjects likely includes both physiological changes and methodological differences in the various studies. Alterations in pulmonary permeability, dilution of intrapulmonary concentrations due to an increased ELF volume, and/or disruption in transport systems in the lungs because of injury or infection have been suggested as physiological explanations for lower ELF concentrations in the critically ill patient (28). Methodological issues that differ between our study and those conducted in critically ill patients included the dose of meropenem administered, volume of lavage fluid instilled (e.g., BAL of $200 \mathrm{ml}$ versus mini-BAL of $40 \mathrm{ml}$ ), analytical assays for urea (to determine dilution factor of pulmonary samples), use of stabilizing solution (e.g., MOPS) for our sample collection, number and timing of measurable ELF concentrations, and reporting of measured versus simulated concentrations (based on population pharmacokinetic analyses). Confirmation of our pharmacokinetic findings in critically ill patients will permit appropriate translation of the observed concentrations of meropenem and RPX7009 in healthy subjects.

In summary, the results of this study provide critical information on the time course and magnitude of plasma and ELF concentrations of following the fixed-dose combination of meropenem-RPX7009. Intravenous administration of meropenem 2 g/RPX7009 $2 \mathrm{~g}$, as a 3-h i.v. infusion for three doses, produced ELF concentrations that ranged from approximately one-half to two times the simultaneous plasma concentrations during the $8 \mathrm{~h}$ dosing interval. The ratios of ELF to plasma concentrations based on the mean $\mathrm{AUC}_{0-8}$ values were 0.63 and 0.53 for meropenem and RPX7009, respectively. The in vitro activity against Gram-negative pathogens, including CRE, and the sustained ELF concentrations above the current MIC breakpoint value of $1 \mu \mathrm{g} / \mathrm{ml}$ for meropenem against Enterobacteriaceae suggest that meropenemRPX7009 has the potential to be an effective agent for the treatment of hospital-acquired and ventilator-associated bacterial pneumonia due to meropenem resistant, mereopenem-RPX7009 susceptible pathogens. The results of the ongoing clinical trials will provide support to whether meropenem-RPX7009 is a suitable agent for the treatment of severe lower respiratory tract infections due to these pathogens.

\section{ACKNOWLEDGMENTS}

This study was supported in part by a research study grant from The Medicines Company. This project has been funded in part with federal funds from the Department of Health and Human Services; the Office of the Assistant Secretary for Preparedness and Response; and the Biomedical Advanced Research and Development Authority (BARDA), under contract no. HHSO100201400002 with Rempex Pharmaceuticals, a wholly-owned subsidiary of The Medicines Company.

E.W. is an investigator for ongoing research studies of The Medicines Company. J.S.L., S.D., D.C.G., and M.N.D. are current employees of Rempex Pharmaceuticals, a wholly owned subsidiary of The Medicines Company. K.A.R. has been a consultant and speaker for The Medicines Company.

\section{REFERENCES}

1. Spellberg B, Bartlett JG, Gilbert DN. 2013. The future of antibiotics and resistance. N Engl J Med 368:299-302. http://dx.doi.org/10.1056 /NEJMp1215093.

2. Livermore DM. 2011. British Society for Antimicrobial Chemotherapy Working Party on The Urgent Need: Regenerating Antibacterial Drug Discovery and Development. Discovery research: the scientific challenge of finding new antibiotics. J Antimicrob Chemother 66:1941-1944.

3. Nordmann P, Naas T, Poirel L. 2011. Global spread of carbapenemaseproducing Enterobacteriaceae. Emerg Infect Dis 17:1791-1798. http://dx .doi.org/10.3201/eid1710.110655.

4. Falagas ME, Lourida P, Poulikakos P, Rafailidis PI, Tansarli GS. 2014. Antibiotic treatment of infections due to carbapenem-resistant Enterobacteriaceae: systematic evaluation of the available evidence. Antimicrob Agents Chemother 58:654-663. http://dx.doi.org/10.1128/AAC.01222-13.

5. U.S. Department of Health and Human Services/Centers for Disease Control and Prevention. 2013. Antibiotic resistance threats in the United States, 2013. Centers for Disease Control and Prevention, Atlanta, GA. http://www.cdc.gov/drugresistance/threat-report-2013/pdf/ar-threats-20 13-508.pdf

6. Drawz SM, Papp-Wallace KM, Bonomo RA. 2014. New $\beta$-lactamase inhibitors: a therapeutic renaissance in an MDR world. Antimicrob Agents Chemother 58:1835-1846. http://dx.doi.org/10.1128/AAC.00826-13.

7. Olsen I. 2015. New promising $\beta$-lactamase inhibitors for clinical use. Eur J Clin Microbiol Infect Dis 34:1303-1308. http://dx.doi.org/10.1007 /s10096-015-2375-0.

8. Toussaint KA, Gallagher JC. 2015. $\beta$-lactam/ $\beta$-lactamase inhibitor combinations: from then to now. Ann Pharmacother 49:86-98. http://dx.doi .org/10.1177/1060028014556652.

9. Hecker SJ, Reddy KR, Totrov M, Hirst GC, Lomovskaya O, Griffith DC, King P, Tsivkovski R, Sun D, Sabet M, Tarazi Z, Clifton MC, Atkins K, Raymond A, Potts KT, Abendroth J, Boyer SH, Loutit JS, Morgan EE, Durso S, Dudley MN. 2015. Discovery of a cyclic boronic acid $\beta$-lactamase inhibitor (PRX7009) with utility versus class A serine carbapenemases. J Med Chem 58:3682-3692. http://dx.doi.org/10 .1021 /acs.jmedchem.5b00127.

10. Castanheria M, Becker HK, Rhomberg PR, Jones RN. 2014. Effect of the $\beta$-lactamase inhibitor RPX7009 combined with meropenem tested against a large collection of KPC-producing Enterobacteriaceae, abstr C-777. Abstr 54th Intersci Conf Antimicrob Agents Chemother. American Society of Microbiology, Washington, DC.

11. Lapuebla A, Abdallah M, Olafisoye O, Cortes C, Urban C, Quale J, Landman D. 2015. Activity of meropenem combined with RPX7009, a novel $\beta$-lactamase inhibitor, against gram-negative clinical isolates in New York City. Antimicrob Agents Chemother 59:4856-4860. http://dx .doi.org/10.1128/AAC.00843-15.

12. Griffith DC, Rubino CM, Loutit JS, Morgan EE, White D, Dudley MN. 2014. A phase 1 study of the safety, tolerability, and pharmacokinetics of a single dose of the beta-lactamase inhibitor RPX7009 alone, meropenem alone, and both in combination (Carbavance) in healthy adult subjects, abstr F-960. Abstr 54th Intersci Conf Antimicrob Agents Chemother. American Society of Microbiology, Washington, DC.

13. Griffith DC, Rubino CM, Loutit JS, Morgan EE, White D, Dudley MN. 2014. A phase 1 study of the safety, tolerability, and pharmacokinetics of the beta-lactamase inhibitor RPX7009 alone, meropenem alone, and both in combination (Carbavance) TID for 7 days in healthy adult subjects, 
abstr 401. Abstr ID Week. Infectious Diseases Society of America, Arlington, VA.

14. Ambrose PG, Bhavnani SM, Ellis-Grosse EJ, Drusano GL. 2010. Pharmacokinetic-pharmacodynamic considerations in the design of hospitalacquired or ventilator-associated bacterial pneumonia studies: look before you leap! Clin Infect Dis 51(Suppl 1):S103-S110.

15. Rodvold KA, George JM, Yoo L. 2011. Penetration of anti-infective agents into pulmonary epithelial lining fluid: focus on antibacterial agents. Clin Pharmacokinet 50:637-664. http://dx.doi.org/10.2165/11594090 -000000000-00000.

16. Baldwin DR, Honeybourne D, Wise R. 1992. Pulmonary disposition of antimicrobial agents: methodological considerations. Antimicrob Agents Chemother 36:1171-1175. http://dx.doi.org/10.1128/AAC.36.6.1171.

17. Rodvold KA, Gotfried MH, Still JG, Clark K, Fernandes P. 2012. Comparison of plasma, epithelial lining fluid, and alveolar macrophage concentrations of solithromycin (CEM-101) in healthy adult subjects. Antimicrob Agents Chemother 56:5076-5081. http://dx.doi.org/10.1128 /AAC.00766-12.

18. Cockcroft DW, Gault MH. 1976. Prediction of creatinine clearance from serum creatinine. Nephron 16:31-41. http://dx.doi.org/10.1159 /000180580.

19. Rennard SI, Basset G, Lecossier D, O’Donnell KM, Pinkston P, Martin PG, Crystal RG. 1986. Estimation of volume of epithelial lining fluid recovered by lavage using urea as marker of dilution. J Appl Physiol 60: 532-538.

20. Baldwin DR, Wise R, Andrews JM, Ashby JP, Honeybourne D. 1990. Azithromycin concentrations at the sites of pulmonary infection. Eur Respir J 3:886-890.

21. Dandekar PK, Maglio D, Sutherland CA, Nightingale CH, Nicolau DP.
2003. Pharmacokinetics of meropenem 0.5 and 2 g every $8 \mathrm{~h}$ as a 3-hour infusion. Pharmacotherapy 23:988-991.

22. Drusano GL, Hutchison M. 1995. The pharmacokinetics of meropenem. Scand J Infect Dis 96(Suppl):11-16.

23. Peleg AY, Salmon M. 2010. Meropenem, p 500-513. In Grayson ML (ed) Kucer's the use of antibiotics, 6th ed. American Society of Microbiology, Washington, DC.

24. Conte JE, Jr, Golden JA, Kelley MG, Zurlinden E. 2005. Intrapulmonary pharmacokinetics and pharmacodynamics of meropenem. Int J Antimicrob Agents 26:449-456. http://dx.doi.org/10.1016/j.ijantimicag.2005.08 .015 .

25. Allegranzi B, Cazzadori A, Di Perri G, Bonora S, Berti M, Franchino L, Biglino A, Cipriani A, Concia E. 2000. Concentrations of single-dose meropenem (1 g i.v.) in bronchoalveolar lavage and epithelial lining fluid. J Antimicrob Chemother 46:319-322. http://dx.doi.org/10.1093/jac/46.2 .319 .

26. Lodise TP, Sorgel F, Melnick D, Mason B, Kinzig M, Drusano GL. 2011. Penetration of meropenem into epithelial lining fluid of patients with ventilator-associated pneumonia. Antimicrob Agents Chemother 55: 1606-1610. http://dx.doi.org/10.1128/AAC.01330-10.

27. Frippiat F, Musuamba FT, Seidel L, Albert A, Denooz R, Charlier C, Van Bambeke F, Wallemacq P, Descy J, Lambermont B, Layios N, Damas P, Moutschen M. 2015. Modeled target attainment after meropenem infusion in patients with severe nosocomial pneumonia: the PROMESSE study. J Antimicrob Chemother 70:207-216. http://dx.doi .org/10.1093/jac/dku354.

28. Felton TW, McCalman K, Malagon I, Isalska B, Whalley S, Goodwin J, Bentley AM, Hope WW. 2014. Pulmonary penetration of piperacillin and tazobactam in critically ill patients. Clin Pharmacol Ther 96:438-448. http://dx.doi.org/10.1038/clpt.2014.131. 